\title{
Problemas percibidos y concepciones de extensión de los técnicos del Centro Nacional de Tecnología Agropecuaria y Forestal de El Salvador, Centroamérica
}

Problemas percebidos e concepções de extensão dos técnicos do Centro Nacional de Tecnologia Agropecuária e Forestal de El Salvador, América Central

\section{Perceived problems and conceptions of extension of the practitioners of the Centro Nacional de Tecnología Agropecuaria y Forestal, El Salvador, Central America}

Les problèmes perçus et les conceptions de la extension de techniciens du Centre National de technologie agricole et des forêts en El Salvador, en Amérique Centrale

Fernando Landini*

(landini_fer@hotmail.com)

Recebido em 02/04/2014; revisado e aprovado em 14/07/2014; aceito em 12/11/2014 DOI: http://dx.doi.org/10.1590/151870122015219

\begin{abstract}
Resumen: La extensión rural se está reposicionando en América Latina como herramienta de desarrollo. 34 extensionistas rurales de El Salvador fueron encuestados para identificar los problemas a los que se enfrentan y la concepción de extensión rural con que guían su práctica. Los encuestados describen como principales problemas el uso de tecnologías inapropiadas, la falta de créditos y las dificultades para el trabajo grupal. A nivel general se observa la presencia de una concepción de la extensión fuertemente transferencista.

Palabras clave: Extensión rural. Desarrollo rural. Transferencia de tecnología.

Resumo: A extensão rural está reposicionando-se na América Latina como ferramenta de desenvolvimento. 34 extensionistas rurais de El Salvador foram pesquisados para identificar os problemas que eles enfrentam e a concepção de extensão rural que orientam a sua prática. Os participantes descreveram como principais problemas o uso de tecnologias inadequadas, a falta de crédito e as dificuldades para o trabalho em grupo. Em geral, os extensionistas pesquisados têm uma concepção fortemente transferencista da extensão rural.

Palavras-chave: Extensão rural. Desenvolvimento rural. Transferência de tecnologia.

Abstract: In Latin America, the use of rural extension as a development tool is regaining acknowledgement. 34 practitioners were surveyed with the aim of identifying the problems faced by Salvadorian rural extensionists, as well as the conception of rural extension that they use to guide their practice. The fundamental problems that the participants describe are the farmers' use of unsuitable technologies, lack of credits and the difficulties of cooperative work. In general terms, the interviewees support a diffusionist approach to rural extension.

Key words: Rural extension. Rural development. Transfer of technology.

Résumé: La vulgarisation rurale redevient en Amérique Latine un outil de développement. 34 «vulgaristes» ruraux du El Salvador ont été interrogés pour identifier les problèmes qu'ils rencontrent et la conception de l'extension rurale qui guide leur pratique. Les enquêtés décrivent comme problèmes principaux l'usage de technologies inappropriées, le manque de crédits et les difficultés pour le travail en groupe. De façon générale, on observe la présence d'une conception de la vulgarisation fortement orientée vers le transfert de technologies

Mots clés: Vulgarisation rurale. Développement rural. Transfert de technologie.
\end{abstract}

\section{Introducción}

Existe creciente interés en América Latina por reposicionar a la extensión como herramienta de desarrollo rural (AGUIRRE, 2012) en el contexto de la revalorización de las políticas de desarrollo activas (HAUSMANN; RODRIK, 2005). Los sistemas de extensión rural latinoamericanos nacen hacia mediados del siglo pasado inspirados en el modelo de extensión estadounidense (DA ROS, 2012; SCHALLER, 2006). Desde fines de los 80s y durante los 90s, la consolidación de las políticas neoliberales, las cuales sostenían la idea de un 'estado mínimo' como premisa para el desarrollo, llevó al desmantelamiento y a la búsqueda de privatización de muchos de los sistemas de extensión del continente (ARDILA, 2010; DIESEL et al., 2008). Con el nuevo siglo, en la mayor parte de América Latina los supuestos neoliberales y las políticas de ajuste estructural empiezan a ser puestas en cuestión, lo que invita a la recuperación de la extensión como instrumento de desarrollo rural (RIVERA, 2002; SELIS, 2012). En El Salvador, la aplicación de políticas de

* Consejo Nacional de Investigaciones Científicas y Técnicas (CONICET), Universidad de Buenos Aires y Universidad de la Cuenca del Plata, Argentina. 
ajuste, fundamentalmente durante los 90s, llevó a una caída drástica del presupuesto del Centro Nacional de Tecnología Agropecuaria y Forestal (CENTA), principal institución de extensión del país, sin que hasta la fecha el nivel de gasto se haya restablecido como porcentaje del gasto público (ARDILA, 2010).

En el contexto de la recuperación de la extensión rural en América Latina en general y en El Salvador en particular, resulta de interés identificar y estudiar los problemas a los que se enfrenta la extensión rural en el país, particularmente el CENTA, con el fin de diseñar e implementar acciones para afrontarlos. En Latinoamérica la investigación sobre extensión rural resulta particularmente escasa (MÉNDEZ, 2006), especialmente si se la compara con la cantidad de publicaciones en temas relacionados, como es el caso de la agricultura familiar.

La mayor parte de las investigaciones destinadas a abordar la temática de la extensión rural se apoyan en estudios de caso puntuales. Haciendo una lectura transversal de estos trabajos se observan múltiples problemas que tienen que ser enfrentados por los extensionistas. Entre ellos pueden mencionarse la escasa adopción de tecnologías por parte de los productores (GADEA, 2009), la dificultad para lograr la participación de los beneficiarios en distintos proyectos (BOAS; GOLDEY, 2005), la falta de adecuación de las planificaciones a las necesidades de los productores (AQUINO; TEIXEIRA, 2005; MURILLO; MARTÍNEZ, 2010), y las trabas para comercializar la producción campesina junto a los bajos precios de venta (ESCOBAR, 2012).

Uno de los problemas más mencionados por los investigadores es la persistencia de enfoques de extensión rural tradicionales, de carácter difusionista (SÁNCHEZ CADENA, 2011; SARAIVA; CALLOU, 2009; SELIS, 2012), aún en contra de los nuevos abordajes recomendados por la academia, por diferentes organismos internacionales y por las mismas instituciones de extensión. La extensión rural tradicional, nacida en los Estados Unidos el siglo pasado, se caracteriza por estructurarse a partir de la transferencia (también denominada 'difusión') de conocimientos 'modernos' desarrollados por los investigadores a los productores del campo
(LANDINI; MURTAGH; LACANNA, 2009). De esta manera, la extensión queda organizada como un vínculo vertical, en donde tanto el saber como el poder quedan ubicados en un polo de la relación, en detrimento de los productores (FREIRE, 1973). En contraste, las propuestas de extensión actualmente se orientan hacia modelos de trabajo participativos (MEDEIROS; BORGES, 2007) centrados en la comunicación y el diálogo horizontal entre técnicos, productores e incluso otros actores que forman parte de los sistemas de innovación agrícola (SOUZA; GOMES, 2008; ZUIN; ZUIN; MANRIQUE, 2011). No obstante, queda claro que el cambio de un modelo vertical-difusionista a uno horizontalparticipativo constituye un gran desafío, ya que se observan importantes problemas para llevar estos cambios a nivel de las prácticas de los extensionistas (LANDINI, 2012; SELIS, 2012; SOUZA; GOMES, 2008).

Así, atendiendo al conjunto de problemas que deben enfrentar los extensionistas y los sistemas de extensión rural, en este trabajo se propone identificar y analizar los problemas enfrentados por los técnicos del CENTA y las concepciones de extensión a partir de las cuales éstos guían sus prácticas, con el fin de aportar al fortalecimiento de la extensión rural salvadoreña. En este sentido, se asume que un mayor conocimiento y comprensión de las problemáticas que limitan su impacto puede contribuir a generar acciones y estrategias que permitan afrontarlas.

\section{Materiales y métodos}

Para alcanzar estos objetivos se realizó una investigación descriptiva basada en la implementación de una encuesta a extensionistas del CENTA. Se destaca que algunos de los resultados desarrollados en este trabajo han sido presentados parcialmente como parte de un comparativo latinoamericano, pero sin que se haya focalizado en el caso salvadoreño (LANDINI, 2013a, en prensa).

A nivel latinoamericano las encuestas fueron realizadas entre los años 2010 y 2013. Las encuestas de El Salvador son del 2010. La encuesta fue enviada y recibida por email. La respuesta fue voluntaria. Se recibieron los formularios de 34 extensionistas de la institución. La cantidad de extensionistas que trabajan en 
el CENTA no pudo ser obtenida ni pudo hacerse una estimación que contara con suficiente fundamento. En consecuencia, dado que se desconocía el total de extensionistas así como su distribución territorial, y que se iba a trabajar con respuestas voluntarias, el tamaño de la muestra no fue calculado estadísticamente como suele hacerse en las investigaciones de tipo cuantitativo (ARGIBAY, 2009). De esta manera, los resultados no deben considerarse como representativos de la totalidad de la extensión rural del CENTA, sino tenidos como expresión de un estudio con énfasis hermenéutico/interpretativo (GUBA; LINCOLN, 1994), cercano a la tradición cualitativa, aun cuando sus resultados se expresen de manera cuantificada.

Las preguntas cerradas de la encuesta se orientaron a relevar información sociodemográfica, mientras que las abiertas abordaron los problemas enfrentados en el trabajo de extensión y las contribuciones de la psicología al trabajo de los extensionistas (ver Anexo). Las respuestas de los encuestados son tomadas en dos sentidos. Primero, como fuente de información sobre una realidad que conocen de primera mano (esto referido fundamentalmente a los problemas relacionados con el trabajo de extensión). Segundo, como indicadores del modo en que los encuestados miran ciertos ámbitos de la realidad, en este caso su concepción de extensión.

Para el análisis de las respuestas se utilizaron los principios generales de la Teoría Fundamentada (DANTAS et al., 2009; LEITE et al., 2012). En concreto, se procedió a la lectura de las respuestas haciendo foco en cada uno de los ejes de análisis indicados. En ese proceso se fueron construyendo categorías, las cuales fueron utilizadas para clasificar los diferentes fragmentos de las respuestas con el apoyo del software Atlas Ti. Luego se realizaron lecturas adicionales del material con el fin de ajustar los contenidos de cada categoría, uniéndose aquellas de límites difusos o separando aquellas que tenían contenidos con identidad propia. Luego de varias lecturas se llegó a una definición final de cada categoría que contuviera criterios de inclusión/exclusión de fragmentos. Como criterio general, durante el proceso de clasificación se optó por incluir los fragmentos que podían presentar dudas, para no cometer el error de excluir aquellos que sí debían ser categorizados. Para terminar, se procedió a una última lectura de los fragmentos incluidos dentro de cada categoría con el fin de excluir aquellos que no correspondieran a su definición final. A nivel operativo, se consideró que una categoría (que expresaba una creencia o afirmación particular) estaba presente en una encuesta cuando había sido mencionada al menos una vez. En las tablas de resultados sólo se presentan aquellas categorías que aparecen en al menos el 25\% de las encuestas. Respeto del eje de análisis correspondiente a los problemas relacionados con el trabajo de extensión sólo se consideraron las preguntas 1 y 2. Respecto de la concepción de extensión se tomaron todas las respuestas (ver el listado de preguntas en el Anexo).

\section{Resultados y discusión}

\subsection{Descripción general de la muestra}

La muestra está compuesta por 33 hombres y 1 mujer. Llama la atención esta relación ya que si bien ésta no fue seleccionada en términos de azar estadístico, no se introdujo ningún criterio de selección más que la voluntad de responder de los participantes. Esto trae como pregunta si realmente existe un predominio masculino tan fuerte en el CENTA y, en ese caso, cuál será su impacto en las actividades que implementa la institución, siendo el más probable la priorización de las prácticas productivas consideradas tradicionalmente masculinas como objeto del trabajo de extensión. Respecto del nivel educativo, el $78 \%$ posee título universitario, mientras que el resto terciarios no universitarios. En ningún caso se indicaron estudios de postgrado, algo frecuente en otros países latinoamericanos como la Argentina por ejemplo, donde más del $40 \%$ de los extensionistas ha realizado cursos de postgrado, especializaciones o maestrías (LANDINI, 2013b), pero no tanto en otros como Paraguay, donde este porcentaje no llega al 10\% (LANDINI, 2013c). Respecto de los 24 casos en los que se indicó una formación universitaria específica, 23 son ingenieros agrónomos y 1 posee título de docente universitario. Si bien cierto predominio de ingenieros agrónomos es usual dentro del personal de extensión 
(LANDINI; BIANQUI, 2014), en este caso la tendencia es sin dudas extrema. Ahora bien, teniendo en cuenta que la extensión rural no sólo requiere de conocimientos técnicos para su implementación efectiva (BOCCHICCHIO, 2013; LANDINI, 2012), sino también de capacidades vinculadas con la gestión de grupos, la implementación de dinámicas participativas y el manejo de procesos de enseñanzaaprendizajes (LANDINI et al., 2009), resulta preocupante que la institución no cuente con profesionales que tengan título relacionado con dichas áreas.

La edad de los encuestados va de los 25 a los 59 años, con una media de 41,33 y un desvío estándar de 9,86. La experiencia de trabajo como extensionistas va de los 2 a los 31 años, con una media de casi 14 años y un desvío estándar de 9,54. Aquí llama la atención que el $38 \%$ de los encuestados tenga una experiencia de entre 2 y 4 años, mientras que el resto se ubique entre los 14 y los 31, quedando un bache de experiencia entre los 5 y los 13 años. Teniendo en cuenta que una amplia mayoría de los extensionistas trabajaron sólo en instituciones gubernamentales, este resultado podría estar sugiriendo la existencia de un período sin contrataciones entre el año 1997 y el 2005, coincidiendo a grandes rasgos con el período 1999-2004, en el cual el PBI de El Salvador permaneció estacado (HAUSMANN; RODRIK, 2005).

\subsection{Problemas vinculados con el trabajo de extensión}

En el Tabla 1 se presentan los problemas que deben enfrentar los extensionistas encuestados en su trabajo. Se indica la cantidad de encuestas en las que éstos fueron mencionados así como el porcentaje que representan del total de participantes.

Tabla 1 - Problemas para el desarrollo y el trabajo de extensión

\begin{tabular}{lcc}
\multicolumn{1}{c}{ Contenidos } & Menciones & \% \\
\hline 1. Productores con tecnologías o manejos productivos inadecuados & 16 & 47,06 \\
\hline 2. Falta de créditos para la producción & 15 & 44,12 \\
\hline 3. Individualismo, desconfianza y falta de asociaciones de productores & 15 & 44,12 \\
\hline 4. Bajo nivel escolar de los productores & 13 & 38,24 \\
\hline 5. Falta de capital, inversión o infraestructura predial & 13 & 38,24 \\
\hline 6. Falta de tierra, tierra improductiva o problemas de tenencia & 12 & 35,29 \\
\hline 7. Problemas para comercializar, bajos precios de venta & 12 & 35,29 \\
\hline 8. Resistencia al cambio y a la adopción de tecnologías & 11 & 32,35 \\
\hline 9. Falta de apoyo del estado y de las instituciones públicas a los productores & 10 & 29,41 \\
\hline 10. Insumos para la producción caros, altos costos & 10 & 29,41 \\
\hline 11. Productores con actitud asistencialista, oportunista, pasivos, desanimados & 9 & 26,47 \\
\hline 12. Productores con escasa diversidad productiva en sus predios & 9 & 26,47 \\
\hline 13. Proyectos e iniciativas de desarrollo diseñados sin la participación de los & 9 & 26,47 \\
\hline productores o que no se adecuan a los territorios & 9 & \multirow{2}{*}{. } \\
\hline
\end{tabular}

A continuación reflexiona sobre estos problemas. No obstante, se deja para subtítulos posteriores el análisis de las implicaciones de este encuadre de problemas, el cual revela mucho de la concepción de extensión de los encuestados (LANDINI et al., 2014).

El más mencionado corresponde a una crítica a las prácticas productivas de los beneficiarios, lo que se expresa en las encuestas como: "producción de secano y uso de tecnologias tradicionales", "mal uso del recurso suelo" o "uso indiscriminado de agroquímicos". Esto se relaciona también con la escasa diversidad productiva, lo que incluye referencias críticas al monocultivo: "la agricultura del pequeño agricultor es tradicional [...] encajonándose en la mayoría de los casos en la producción de granos básicos". A la par, al señalarse el "tradicionalismo" de los productores, también se plantea que estas prácticas productivas constituyen trabas para la adopción de nuevas tecnologías a partir del "arraigamiento de los patrones culturales de parte de los productores", lo que lleva a la "falta de adopción de tecnologías a nivel familiar".

En segundo lugar aparece la falta de créditos para la producción, problema que se articula con la falta de capital y de infraestructura predial de los productores. Estos 
problemas, sumados a la falta de tierra o a los problemas de tenencia, nos retrotraen al rol que jugó la cuestión de la tierra en la guerra civil salvadoreña y en los acuerdos de paz posteriores (KAY, 2003), en los cuales la entrega de tierras generó a los beneficiarios una deuda que en la mayoría de los casos se les hizo imposible de pagar (BREMOND, 2007). Si bien hacia fines de los 90 esa deuda fue condonada casi en su totalidad, los resultados de la encuesta sugieren la gran importancia que conservan estos problemas. A la vez, en el caso de la falta de crédito, hay que hacer referencia al impacto de los políticas de ajuste estructural de los 90, las cuales incluyeron la privatización de la banca pública, con las consecuencias que un encuestado menciona: "la privatización de la banca nacional hizo muy difícil el acceso a créditos para el pequeño productor al no tener las garantías".

En tercer lugar aparece como problema el individualismo, la desconfianza y la falta de asociaciones de productores. Esto se expresa como "falta de organización" por parte de los productores o como "conductas individualistas (falta de confianza, egoísmo)". Un encuestado lo analiza de esta manera: "la mayoría de los proyectos vienen formulados para trabajar en forma asociada, lo que lleva a la dificultad en muchos de los casos de encontrarse con muchos conflictos internos dentro de estas organizaciones". Como se mencionaba anteriormente, los extensionistas deben gestionar trabajos grupales en el contexto de su función. Asumiento que su formación es técnica, no social, no resulta extraña la presencia de este problema.

Por su parte, el "bajo nivel educativo" de los productores, así como el "alto nivel de alfabetismo", también son descriptos como problemas. Si bien esto no es aclarado en las encuestas, posiblemente se relacione con la dificultad que encuentran los técnicos a la hora de formar o capacitar a los productores por la existencia de limitaciones para seguir argumentos técnicos, como han sugerido diferentes autores (GAITÁN; PACHÓN, 2010; HENZ, 2010).

Otra de las dificultades mencionadas, en este caso por poco más de un tercio de los encuestados, son los "problemas para comercializar su producción", lo que incluye "los malos precios de venta de sus cosechas".
Esto es explicado por "desconocimiento de mercadeo", "utilización de intermediarios para la venta de producción" o por "invasión de mercadería de los países vecinos", esto último pudiendo vincularse con el Tratado de Libre Comercio entre EEUU, Centroamérica y República Dominicana (CAFTA), que tiene en los agricultores uno de los actores más golpeados (TAYLOR; YÚNEZ; JESURUN-CLEMENTS, 2010).

La falta de apoyo del estado a los productores, por su parte, hace referencia a la "falta de políticas que impulsen a la agricultura" o a "cero políticas de apoyo a los productores para poder desarrollarse de parte de los gobiernos". En definitiva, se argumenta que "no exiten políticas adecuadas de protección e incentivos a pequeños y medianos productores agropecuarios". Indudablemente, estas dificultades pueden reconducirse a las políticas de ajuste estructural y de liberalización del comercio implementadas a partir de los años 90, las cuales llevaron a la privatización de diferentes sectores de la economía y a la retirada del Estado como constructor de políticas activas de desarrollo. Como señala un encuestado, estas políticas llevaron a "disminuir la agricultura y a impulsar el comercio en el país", como de hecho se deduce de análisis realizados por diferentes autores (ROBLES, 2010).

Respecto del problema de la 'actitud asistencialista' de los productores, se lo abordará más adelante en relación a la concepción de extensión de los encuestados. El último problema mencionado por los técnicos participantes hace referencia a la existencia de proyectos e iniciativas de desarrollo diseñados sin la participación de los beneficiarios. Esto lo explica con claridad un encuestado cuando dice que "ha habido muchos modelos y muchos programas de extensión tanto de ONGs, como del gobierno [...] que han definido sus líneas de acción [...] sin tomar en cuenta el sentir y pensar de los beneficiarios o afectados". Es decir, proyectos diseñaros y/o implementados sin participación de los beneficiarios, problema que también ha sido señalado por autores en otros contextos (MURILLO; MARTÍNEZ, 2010; TAVEIRA; OLIVEIRA, 2008). 
3.3 Concepción de extensión de los encuestados

En el Tabla 2 se mencionan los elementos que dan forma a la concepción de extensión rural de los extensionistas encuestados del CENTA.

Tabla 2 - Concepción de la tarea de extensión rural

\begin{tabular}{lcc}
\hline \multicolumn{1}{c}{ Contenidos } & Menciones & $\%$ \\
\hline $\begin{array}{l}\text { 1. Extensión orientada al cambio de mentalidad, o a la transferencia/ } \\
\text { adopción de tecnologías por parte de los productores }\end{array}$ & 26 & 76,47 \\
\hline 2. Desvalorización del saber o de las prácticas productivas campesinas & 17 & 50,00 \\
\hline 3. Hacer diagnósticos, planificar, dar seguimiento y evaluar proyectos & 17 & 50,00 \\
\hline 4. Formar/gestionar grupos de productores, manejar conflictos & 16 & 47,06 \\
\hline 5. Capacitar, enseñar y formar al productor & 15 & 44,12 \\
\hline $\begin{array}{l}\text { 6. Reconocimiento/valoración de la participación y de tener en cuenta las } \\
\text { necesidades, intereses y racionalidad del productor en el trabajo de extensión }\end{array}$ & 12 & 35,29 \\
\hline 7. Intervenir en genero, juventud y niñez como parte del trabajo de extensión & 10 & 29,41 \\
\hline 8. Concientizar, motivar, hacer entender o hacer cambiar al productor & 9 & 26,47 \\
\hline
\end{tabular}

A los fines analíticos, pueden diferenciarse tres tipos o núcleos de contenidos que dan forma a la concepción de extensión rural de los encuestados. Por un lado están los elementos que configuran (1) una concepción transferencista o difusionista de la extensión, luego se observan diferentes contenidos que describen (2) diferentes actividades propias del trabajo con productores, y finalmente (3) el señalamiento de la importancia de la participación en el trabajo de extensión rural. Con fragmentos en más del $76 \%$ de las encuestas puede observarse una definición de la extensión como cambio de mentalidad o como transferencia/adopción de tecnologías por parte de los productores (extensión difusionista). Respecto de la extensión como cambio de mentalidad, los encuestados hablan de la necesidad de un "cambio de paradigmas, [de] lograr un cambio en [las] actitudes, habilidades y destrezas de la familia rural". Dicho de manera algo más fuerte: "cambiar la extrechez de razocinio de los productores [respecto de] ciertos temas que obstaculisan su progreso". En relación a la idea de transferencia de tecnologías, un encuestado señala que "en algunos casos y zonas, no existe o es mínima la asistencia técnica que facilite la transferencia de tecnología". Finalmente, en otras encuestas también se habla de la "falta de adopción de tecnologías a nivel familiar" o de "adopción limitada de tecnología". De esta manera, se configura una visión de la extensión rural focalizada en el cambio de la 'mentalidad' de los productores (situación que en cierto sentido los toma como 'objetos' del accionar de la extensión), y en la transferencia y adopción de tecnologías. La extensión transferencista es descripta por diferentes autores como un modo tradicional de pensar la extensión, derivado del modelo estadounidense, el cual asume que son los expertos (investigadores, extensionistas) los portadores del único conocimiento legítimo, mientras que los productores son percibidos como portadores de conocimientos inapropiados o 'atrasados' (e.g. LANDINI; MURTAGH; LACANNA, 2009; MACHADO; DE HEGEDÜS; SILVEIRA, 2006; SCHALLER, 2006). Siendo que se asume que el técnico sabe (en un sentido amplio y totalizador) y que el productor no posee conocimientos relevantes, la extensión termina construyéndose como 'transferencia' de quien sabe a quien no sabe (FREIRE, 1973). Asumido esto, no resulta estraño que el 50\% de los encuestados también tenga una visión negativa de los conocimientos y saberes de los productores.

Enfocada la extenisión rural de esta manera, no resulta inesperado que los encuestados perciban 'resistencia al cambio y a la adopción de tecnologías' por parte de los productores. Es que una interpretación del problema en esos términos parte de 
asumir que el productor debería adoptar la tecnología que se le transfiere en tanto se la considera como la mejor para él, sin que se ponga en cuestión si efectivamente lo que se ofrece coincide con los intereses, necesidades y valores de los beneficiarios. En este contexto tampoco es extraño encontrar descripciones de actitudes pasivas o asistencialistas por parte de los productores (planteadas por muchos encuestados como problema), ya que los agricultores no suelen sentirse comprometidos cuando los objetivos de las acciones no coinciden con lo que internamente ellos consideran más importante.

En paralelo, se observa que un porcentaje importante de los encuestados, poco más de un tercio $(35,29 \%)$, hacen referencia a la importancia de la participación y de tener en cuenta las necesidades, intereses y racionalidad del productor en el trabajo de extensión. Podría pensarse que aquellos que no poseen una visión difusionista son los más propensos a valorar la participación. No obstante, este no parece ser el caso, ya que mientras que el 35\% de quienes tienen un enfoque difusionista valoran la participación, el 37\% de quienes no lo tienen también la valoran, siendo la diferencia entre porcentajes bastante pequeña. Este resultado coincide con dos estudios recientes, uno realizado en Argentina (LANDINI, 2015) y otro en Paraguay (LANDINI; BIANQUI; CRESPI, 2013), los cuales señalan que la valoración de la participación no permite diferenciar entre extensionistas que poseen y no poseen abordajes difusionistas, posiblemente porque la valoración de la participación se relacionaría más con la adopción de un discurso valorado socialmente que con un compromiso real con su contenido.

Dentro de los contenidos referidos a la concepción de extensión rural también se observan un conjunto de tareas que hacen a la labor de extensión. Dentro de ellas se destacan el trabajo con el ciclo completo del proyecto (diagnóstico, planificación, seguimiento y evaluación) (50\%), la formación o gestión de grupos (47,06\%) y la capacitación/formación de productores $(44,12 \%)$. Visto esto, resulta interesante mencionar que sólo la capacitación de productores se corresponde de manera directa con la formación técnica de los encuestados, mientras que el diseño de proyectos y el trabajo con grupos aparecen como tareas que no se identifican necesariamente con su formación (particularmente el trabajo con grupos). Por último, también se hace mención del trabajo a nivel de género, juventud y niñez, y de la motivación/concientización de productores, que hasta cierto punto podría vincularse con una mirada difusionista de la extensión.

\section{Reflexiones y síntesis final}

En este trabajo se ha señalado el alto predominio masculino en la muestra de extensionistas del CENTA. Queda abierta la cuestión de si este porcentaje se mantiene entre los extensionistas de la institución y cuáles son sus implicaciones. En cualquier caso, apoyar la equidad de género a nivel de los agricultores familiares también requiere que esta equidad exista a nivel de las instituciones de apoyo.

La formación de los extensionistas encuestados se concentra en el área productiva (fundamentalmente agronómica). Dado que varios de los problemas mencionados van más allá de la formación técnica, y que la función misma del extensionista requiere conocimientos provenientes del ámbito social, parece recomendable plantearse la distribución disciplinar de los extensionistas del CENTA, buscando incorporar profesionales de disciplinas no técnicas. A la vez, en este contexto, la formación de postgrado aparece como una alternativa interesante, particularmente en áreas como la extensión rural, el desarrollo, la comercialización y las ciencias sociales. El mismo CENTA podría apoyar este proceso de formación de su personal. Por su parte, si bien la cuestión de la falta de créditos y el problema del acceso a la tierra exceden lo que está bajo el control de los extensionistas y de la misma institución, parecen ser cuestiones que requieren una atención particular, en especial el acceso a la tierra, teniendo en cuenta el contexto histórico salvadoreño.

Posiblemente, la existencia de una fuerte concepción difusionista de la extensión rural en los encuestados sea el principal problema identificado. Indudablemente, la implementación de estrategias de intervención con estas características está limitando el impacto de las tareas de extensión, lo que puede intuirse al encontrar dentro de los 
problemas presentados por los encuestados algunos potenciados por este tipo de acciones. En relación a esto, resulta fundamental generar estrategias institucionales que permitan repensar estas prácticas y su impacto, para avanzar hacia modalidades de extensión críticas y complejas, más orientadas a generar capacidades que a entregar respuestas definidas de antemano, favoreciéndose así un posicionamiento activo y dinámico de los productores. En este contexto, la implementación de modalidades de capacitación reflexivas puede constituir una alternativa de gran interés (LANDINI; BIANQUI; RUSSO, 2013). A la vez, la formación en extensión y en desarrollo rural a nivel de cursos de capacitación o de postgrado podría constituir una alternativa válida. En cualquier caso, repensar las estrategias de extensión parece una tarea necesaria, si lo que se busca realmente es potenciar y fortalecer la agricultura familiar salvadoreña.

\section{Anexo: Listado de preguntas abiertas incluidas en la encuesta}

1. Según su experiencia ¿cuáles son los principales problemas que tienen los pequeños productores para progresar y salir adelante? 2. Muchas veces los proyectos de extensión y de desarrollo rural con pequeños productores no logran los resultados que se esperan. Además de lo mencionado en la pregunta anterior, ¿qué otras dificultades surgen en el trabajo de extensión?

3 . De los problemas y dificultades mencionados en las preguntas 1 y 2 , ¿usted piensa que la psicología podría ayudar a resolver alguno de ellos? [Si responde SI pase a la pregunta 4, si responde NO pase a la pregunta 5]

4. Si respondió SÍ a la pregunta anterior

4.1. ¿Qué dificultades o problemas mencionados en las preguntas 1 y 2 podría ayudar a resolver la psicología?

4.2. ¿Qué otros aportes podría hacer la psicología al trabajo de extensión?

4.3. Si no lo mencionó en alguna respuesta previa ¿de qué manera piensa que los psicólogos/as podrían hacer estos aportes al trabajo de extensión? ¿en qué actividades o acciones concretas podrían implicarse?

5. Si respondió NO a la pregunta 3, ¿por qué piensa que la psicología no podría ayudar a resolver ninguno de los problemas mencionados?

6. Si desea agregar algún comentario puede hacerlo aquí:

\section{Referencias}

AGUIRRE, Francisco. El nuevo impulso de la extensión rural en América Latina. Situación actual y perspectivas. Santiago de Chile: Red Latinoamericana para los Servicios de Extensión Rural, 2012.

AQUINO, Joacir; TEIXEIRA, Olívio. Agricultura familiar, crédito e mediação institucional: a experiência do PRONAF em São Miguel no Nordeste Brasileiro. Cuadernos de desarrollo rural, Bogotá, v. 2, n. 54, p. 61-85, 2005.

ARDILA, Jorge. Extensión rural para el desarrollo de la agricultura y la seguridad alimentaria: aspectos conceptuales, situación y una visión de futuro. San José, Costa Rica: Instituto Interamericano de Cooperación para la Agricultura, 2010.

ARGIBAY, Juan. Muestra en investigación cuantitativa. Subjetividad y Procesos Cognitivos, Buenos Aires, v. 13, n. 1, p. 13-29, 2009.

BOAS, Ana; GOLDEY, Patricia. A comparison on farmers' participation in farmers' organizations and implications for rural extension in Minas Gerais. Organizações Rurais \& Agroindustriais, Lavras, MG, v. 7, n. 3, p. 259-270, 2005.

BOCCHICCHIO, Ana. Perfil profesional en el área de extensión y desarrollo rural. En: FEITO, María; BATTISTA, Susana; CORIA, Lorena; LANDINI, Fernando (Ed.). Contribuciones internacionales al Desarrollo Local y Rural: hacia una agenda de investigación. San Justo, Argentina: Universidad Nacional de La Matanza, 2013. p. 184-188.

BREMOND, Ariane. The politics of peace and resettlement through El Salvador's land transfer programme: caught between the state and the market. Third World Quarterly, v. 28, n. 8, p. 1537-1556, 2007.

DA ROS, César. Gênese, desenvolvimento, crise e reformas nos serviços públicos de extensão rural durante a década de 1990. Mundo Agrario, La Plata, v. 13, n. 25, sin páginas, 2012.

DANTAS, Claudia; LEITE, Joséte; LIMA, Suzinara; STIPP, Marluci. Teoría fundamentada en los datos. Aspectos conceptuales y operacionales: metodología posible de ser aplicada en la investigación en enfermería. Revista Latino-Americana de Enfermagem, Ribeirão Preto, Brasil, v. 17, n. 4, p. 573-579, 2009.

DIESEL, Vivien; FROEHLICH, José; NEUMANN, Pedro; SILVEIRA, Paulo. Privatização dos serviços de extensão rural: uma discussão (des)necessária? Revista de Economia e Sociologia Rural, Brasília v. 46, n 4, p. 11551188, 2008.

ESCOBAR, Germán. Diseño de una agenda de extensión rural latinoamericana para un desarrollo rural inclusivo. Extensión rural con un enfoque participativo y de mercado: hacia un marco conceptual. Santiago, Chile: Centro Latinoamericano para el Desarrollo Rural, 2012.

FREIRE, Paulo. ¿Extensión o comunicación? La concientización en el medio rural. Buenos Aires: Siglo XXI, 1973. 
GADEA, Rafael. Extensión rural en el Paraguay: experiencias del proyecto de manejo sostenible de los recursos naturales (PMRN). Revista IICA Uruguay, Montevideo, n. 2, p. 8-10, 2009.

GAITÁN, Carlos; PACHÓN, Fabio Causas para la adopción de tecnologías para la renovación de cafetales. Caso El Colegio (Cundinamarca). Agronomía Colombiana, Bogotá, v. 28, n. 2, p. 329-336, 2010.

GUBA, Egon; LINCOLN, Yvonna. Paradigmas en pugna en la investigación cualitativa. En: DENZIN, Norman; LINCOLN, Yvonna (Ed.). Handbook of qualitative research. Londres: Sage, 1994. p. 105-117.

HAUSMANN, Ricardo; RODRIK, Dani. Self-discovery in a development strategy for El Salvador. Economía, Massachusetts, v. 6, n. 1, p. 43-101, 2005.

HENZ, Gilmar. Desafios enfrentados por agricultores familiares na produção de morango no Distrito Federal. Horticultura Brasileira, Brasília, v. 28, n. 3, p. 260-265, 2010.

KAY, Cristóbal. Estructura agraria y violencia rural en América Latina. Sociologias, Porto Alegre, RS, n. 10, p. 220-248, 2003.

LANDINI, Fernando. Problemas en la extensión rural paraguaya: modelos de extensión en la encrucijada. Cuadernos de Desarrollo Rural, Bogotá, v. 9 n. 69, p. 127149, 2012.

Concepción de extensión rural en 10 países Latinoamericanos. Manuscrito enviado para publicación, 2013a.

Perfil de los extensionistas rurales argentinos del sistema público. Mundo Agrario, La Plata, v. 14., n. 27, sin páginas, $2013 \mathrm{~b}$.

Perfil de los extensionistas de la Dirección de Extensión Agraria. Revista Investigación Agraria, Asunción, v. 15, n. 1, p. 33-40, 2013c.

Different Argentine rural extensionists' mindsets and their practical implications. The Journal of Agricultural Education and Extension, Wageningen, Holanda, v. 21, n. 3, p. 219-234, 2015.

LANDINI, Fernando; BIANQUI, Vanina. Latin American rural extensionists' socio-demographic profile. Ciência Rural, Santa Maria, RS, v. 44, n. 3, p. 575-581, 2014.

LANDINI, Fernando: BIANQUI, Vanina; CRESPI, Melina. Evaluación de las creencias sobre extensión rural de los extensionistas paraguayos. Psiencia, Buenos Aires, v. 5, n. 1, p. 3-14, 2013

LANDINI Fernando; BIANQUI, Vanina; RUSSO, Mabel. Evaluación de un proceso de capacitación para extensionistas rurales implementado en Paraguay. Revista de Economia e Sociologia Rural, Brasília, v. 51, n. sup. 1, p. s009-s030, 2013.

LANDINI Fernando; LEEUWIS, Cees; LONG, Norman; MURTAGH, Sofía. Towards a psychology of rural development processes and interventions. Journal of Community and Applied Social Psychology, v. 24, n. 6, p. 534-546, 2014

LANDINI, Fernando; MURTAGH, Sofía; LACANNA, María. Aportes y reflexiones desde la psicología al trabajo de extensión con pequeños productores. Formosa, Argentina: Instituto Nacional de Tecnología Agropecuaria, 2009.
LEITE, Joséte; SILVA, Laura; OLIVEIRA, Rosane; STIPP, Marluci. Thoughts regarding researchers utilizing Grounded Theory. Revista da Escola de Enfermagem da USP, São Paulo, v. 46, n. 3, p. 765-769, 2012.

MACHADO, João; DE HEGEDÜS, Pedro; SILVEIRA, Laurício. Estilos de relacionamento entre extensionistas e produtores. Ciência Rural, Santa Maria, RS, v. 36, n. 2, p. 641-647, 2006.

MEDEIROS, Jássio; BORGES, Djalma. Participação cidadã no planejamento das ações da Emater-RN. Revista de Administração Pública, Rio de Janeiro, v. 41, n. 1, p. 63-81, 2007.

MÉNDEZ, Marlon. Los retos de la extensión ante una nueva y cambiante noción de lo rural. Revista Facultad Nacional de Agronomía, Medellín, v. 59, n. 2, p. $3407-$ 3423, 2006.

MURILLO, Daniel; MARTÍNEZ, Jorge. Comunicación para el desarrollo en México: reflexiones sobre una experiencia en el trópico húmedo. Estudios sobre las Culturas Contemporáneas, Colima, México, v. 16, n. 31, p. 201-225, 2010.

RIVERA, Fernando. Una aproximación al desarrollo rural de Costa Rica. La extensión agrícola: breve síntesis de su evolución y tendencias. Educare, San José, Costa Rica, n. 3, p. 29-43, 2002.

ROBLES, Francisco. Nuevos espacios de acumulación: modelo de ajuste estructural en El Salvador y Costa Rica (1980-1999). Revista Ciencias Sociales, San José, Costa Rica, n. 128-129, p. 97-117, 2010.

SÁNCHEZ CADENA, Dulce. Dimensión informativa vs. dimensión comunicativa en el concepto de extensión. ¿La definición según Swanson en verdad ya está superada? Ra Ximhai, Mochicahui, México, v. 7, n. 3, p. 437-444, 2011

SARAIVA, Rosa; CALLOU, Angelo. Políticas públicas e estratégias de comunicação para o desenvolvimento local de comunidades pesqueiras de Pernambuco. Interações, Campo Grande, MS, v. 10, n. 1, p. 73-81, 2009.

SCHALLER, Nilo. Extensión rural: ¿hacia dónde vamos?, ¿hacia dónde ir? El Colorado, Argentina: Instituto Nacional de Tecnología Agropecuaria, 2006.

SELIS, Dardo. Análisis de la institucionalidad asociada a los procesos de innovación tecnológica en el sector hortícola del Gran La Plata. Mundo Agrario, La Plata, v. 12, n. 24, sin páginas, 2012

SOUZA Irenilda de; GOMES, Ana. Folkcomunicaçao e extensão rural brasileira: as estratégias de comunicação rural para o desenvolvimento local. Razón y Palabra, Monterrey, n. 60, sin páginas, 2008.

TAVEIRA, Luís; OLIVEIRA, Julieta. A extensão rural na perspectiva de agricultores assentados do Pontal do Paranapanema. Revista de Econonomia e Sociologia Rural, Brasília, v. 46, n. 1, p. 9-30, 2008.

TAYLOR, J. Edward; YÚNEZ, Antonio; JESURUNCLEMENTS, Nancy. Does agricultural trade liberalization reduce rural welfare in less developed countries? The case of CAFTA. Applied Economic Perspectives and Policy, Oxford, v. 32, n. 1, p. 95-116, 2010.

ZUIN, Luís; ZUIN, Poliana; MANRIQUE, Miguel. A comunicação dialógica como fator determinante para 
os processos de ensino-aprendizagem que ocorrem na capacitação rural: um estudo de caso em um órgão público de extensão localizado no interior do Estado de São Paulo. Ciência Rural, Santa Maria, RS, v. 41, n. 5, p. 917-923, 2011. 